\title{
Epidemic spreading with awareness diffusion on activity-driven networks
}

\author{
Ping Hu, Li Ding," and Xuming An \\ School of Electrical Engineering and Automation, Wuhan University, Wuhan 430072, P. R. China
}

(Received 19 August 2018; published 28 December 2018)

\begin{abstract}
Acknowledging the significance of awareness diffusion and behavioral response in contagion outbreaks has been regarded as an indispensable prerequisite for a complete understanding of epidemic spreading. Recent studies from the research community have accumulated overwhelming evidence for the incessantly evolving structure of the underlying networks. Thus there is an impelling need to capture the interplay between the epidemic spreading and awareness diffusion on time-varying networks. In this paper, we consider a behavioral model in which susceptible individuals become alert and adopt a preventive behavior under the local risk perception characterized by a decision-making threshold. The impact of awareness diffusion on the epidemic threshold is investigated under the framework of activity-driven network. Results show that the local epidemic situation in risk perception and the duration of preventive effect are crucial for raising the epidemic threshold. The analytical results are corroborated by Monte Carlo simulations.
\end{abstract}

DOI: 10.1103/PhysRevE.98.062322

\section{INTRODUCTION}

The modeling and analysis of epidemic spreading lie at the core of our understanding about the unfolding of dynamical processes in complex networks, such as malware spreading and information dissemination. In spite of the major advances in this area, one common assumption has been employed that considers a time separation between the evolution in network structures and the contagion process, which limits the spreading process to be typically considered on static networks or annealed networks [1-3]. As the latest empirical studies showed that the contagion of many infectious diseases and the evolution of network structure have a comparable time scale $[4,5]$, the theoretical analysis of these contagion processes requires the development of a novel analytical framework. Correspondingly, an activity-driven approach has been recently put forward for an explicit representation of time-varying connectivity patterns [6]. Under the framework of activity-driven networks (ADNs), the epidemic models incorporating other network characteristics have been proposed by adapting the generative rules of ADNs, such as the community structure [7-9] and the metapopulation model [10], the lifetimes of links [11] and weak/strong ties [12,13], the node's attractiveness $[14,15]$ and the mutual selection [16].

The above works are concerned about the influence of network structure on the epidemic spreading process. Furthermore, nodes in the network can only be passively infected. However, in the real world, individuals are in the position to be aware of the potential threat of disease and they would behave accordingly. Remarkably, risk perception of infectious disease has been acknowledged as an indispensable ingredient for accurate modeling of epidemic spreading process on static networks [17]. In this context, the awareness is reflected in the contact behavior that would change the connection patterns

\footnotetext{
*liding@whu.edu.cn
}

$[18,19]$, or the preventive measures that would modify the propagation parameters $[20,21]$. Inspired by them, there is an impelling need to include the individual's behavior for depicting contagion processes on the ADNs. Among the limited works in this direction, authors in Ref. [22] investigated the effect of human behavioral adaptations by assuming that the activity level of individuals would be reduced based on their own states and global awareness effect. Such an activity reduction is also considered in Ref. [23], with the risk perception embodied in a function of the global prevalence or the growth rate of infection. In addition to the reduction of activity level in contact behavior, the preventive behaviors that would decrease the risk of infection without disrupting the connection patterns is considered in a recent study [24].

A common feature of the risk perception among the existing works is that the effect of awareness is specified by the time-varying contact or infection rate, which is actually limited in properly modeling the underlying decision process [25]. A more practical phenomenon is that the preventive behavior is adopted once the individual's risk awareness reaches or exceeds a critical value [26,27]. In this case, the effect of awareness is embodied in the adoption of selfprotecting behavior, which could then be better described by a threshold model [28]. Particularly, the threshold model has gained prominence in characterizing complex contagion phenomenons in which multiple sources of exposure are required for the adoption [29], such as the spread of fads and ideas over static networks $[30,31]$ and temporal networks [32,33]. To the best of our knowledge, the risk perception characterized by a decision-making threshold has not been included in previous studies under the framework of ADNs yet.

Furthermore, all these risk perceptions in ADNs are based on the current local or global infection prevalence, and thus individual's behavioral decision is only subject to the infectious population. The herd behavior that people would like to follow those who take precautionary measures to protect themselves has been observed to be one of the most common 
social behaviors, and has recently attracted substantial attentions from the research field [34-36]. In this way, the risk awareness of infection diffuses among the susceptible population and consequentially influences their decision making processes. Or rather, the number of alert neighbors in the local epidemic situation has a profound impact on suppressing epidemic explosion. Nevertheless, the spread of awareness is rarely considered in the contagion process on ADNs, and its modeling and analysis are still challenging as a consequence of the incessantly evolving interaction patterns.

To this end, we consider a threshold model for the individual's risk perception in ADNs. The threshold is defined as a critical value beyond which individuals would take selfprotecting measures and thus gain a lower risk of infection. Specifically, in order to circumvent the limit of node's inactivity in ADNs under which the instantaneous neighbors of an inactive node might be too few to make a decision, we only consider the absolute threshold model throughout the paper, which means the risk perception is based on the absolute number of the infectious or alert neighbors. An analytically tractable epidemic model in the ADNs is formulated that explicitly incorporates the typical susceptible-infectedsusceptible (SIS) epidemic spreading with the awareness diffusion characterized by the threshold model. The effects of risk perception and awareness diffusion on the epidemic threshold are investigated by both theoretical analysis and numerical simulations. It is found that the number of alert neighbors in local epidemic situation and the duration of preventive effect are critical for restraining epidemic outbreak: (i) If the risk perception is only based on the number of infectious neighbors, the epidemic threshold remains unchanged when the preventive effect is not sustainable, which will be increased with the permanent effect. (ii) When the number of alert neighbors are considered in the risk perception, the epidemic threshold will be enhanced with the rapid awareness diffusion.

The rest of this paper are organized as follows. In Sec. II, an epidemic model including the indirect feedback loop between individual's behavior and epidemic spreading on the ADNs is established. Theoretical analysis of the epidemic threshold is carried out in Sec. III. In Sec. IV, numerical simulations are conducted to verify the presented theoretical results. Conclusions are drawn in Sec. V.

\section{MODEL}

This section introduces the ADNs and the epidemiological model.

\section{A. Activity-driven network}

We consider the activity-driven network model proposed in Ref. [6]. In this model, each node (individual) $i$ in a population of $N$ nodes is assigned to an activity potential $a_{i}$, which is defined as the probability to create connection with other nodes per unit time. $a_{i}$ is a random variable extracted from a given activity probability distribution $F(a)$. The instantaneous network $G_{t}$ is generated as follows. At each time step $t, G_{t}$ starts with $N$ disconnected nodes. Each node $i$ is activated with probability $a_{i}$ and then generates $m$ edges randomly connected to $m$ other nodes. All these edges in $G_{t}$ would be deleted at next time step $t+\Delta t$ and the procedure is iterated. Without loss of generality, we set $\Delta t=1$ in the following.

For the latter modeling of the epidemic spreading process, the degree distribution and the connected activity-activity correlation of the instantaneous network $G_{t}$ should be derived first. Similar to the heterogeneous mean-field approach for static networks, we group nodes into different classes according to their activities. Thus, all nodes with the same activity belong to the same class and are considered statistically equivalent. At each time step $t$, the inactive nodes have the possibility to be passively connected by the active nodes. Given the activity potential distribution $F(a)$ and the definition of $m$, it is expected that $m\langle a\rangle N$ edges exist in $G_{t}$, where $\langle a\rangle=\sum_{a} a F(a)$. Then we get the probability $P_{I}(k)$ that an inactive node has degree $k$ [7]

$$
P_{I}(k)=\left(\begin{array}{c}
m\langle a\rangle N \\
k
\end{array}\right)\left(\frac{1}{N}\right)^{k}\left(1-\frac{1}{N}\right)^{m\langle a\rangle N-k} .
$$

In the thermodynamic limit, i.e., $N \rightarrow \infty$, the Poisson theorem gives

$$
P_{I}(k)=\frac{(m\langle a\rangle)^{k}}{k !} e^{-m\langle a\rangle} .
$$

For an active node with degree $k$, the number of edges emitted from it and that received from other nodes are expected to be $m$ and $k-m$, respectively. Thus the probability $P_{A}(k)$ that an active node has degree $k$ is

$$
P_{A}(k)= \begin{cases}0 & k<m, \\ \frac{(m\langle a\rangle)^{k-m}}{(k-m) !} e^{-m\langle a\rangle} & k \geqslant m .\end{cases}
$$

Combining Eqs. (2) and (3), the instantaneous degree distribution $P_{a}(k)$ for the nodes with activity $a$ in network $G_{t}$ is given by

$$
P_{a}(k)=(1-a) P_{I}(k)+a P_{A}(k) .
$$

We next discuss the connected activity-activity correlation of instantaneous network $G_{t}$ that is defined as a preference for one node to attach to others with similar activity. Analogous to the connected degree-degree correlation in the static networks [37], we define $F\left(a, a^{\prime}\right)$ as the probability of finding a node with activity $a$ and another node with activity $a^{\prime}$ at the two ends of a randomly selected edge in $G_{t}$. Obviously, $F\left(a, a^{\prime}\right)$ equals to the ratio of the number of edges between nodes with activity $a$ and nodes with activity $a^{\prime}$, to the number of edges in $G_{t}$. Also, $F_{n}(a)$ is defined as the distribution of the remaining activity denoting the probability that there is a node with activity $a$ at the end of a randomly selected edge in $G_{t}$. Following the generation rules of $G_{t}$, we have

$$
\begin{gathered}
F\left(a, a^{\prime}\right)=\frac{N m\left(a+a^{\prime}\right) F(a) F\left(a^{\prime}\right)}{2 m\langle a\rangle N}=\frac{\left(a+a^{\prime}\right) F(a) F\left(a^{\prime}\right)}{2\langle a\rangle}, \\
F_{n}(a)=\frac{N m(a+\langle a\rangle) F(a)}{2 m\langle a\rangle N}=\frac{(a+\langle a\rangle) F(a)}{2\langle a\rangle} .
\end{gathered}
$$

It can be easily proved that the quantity $F\left(a, a^{\prime}\right)$ obeys the sum rules of $\sum_{a, a^{\prime}} F\left(a, a^{\prime}\right)=1$ and $F_{n}(a)=\sum_{a^{\prime}} F\left(a, a^{\prime}\right)$. 


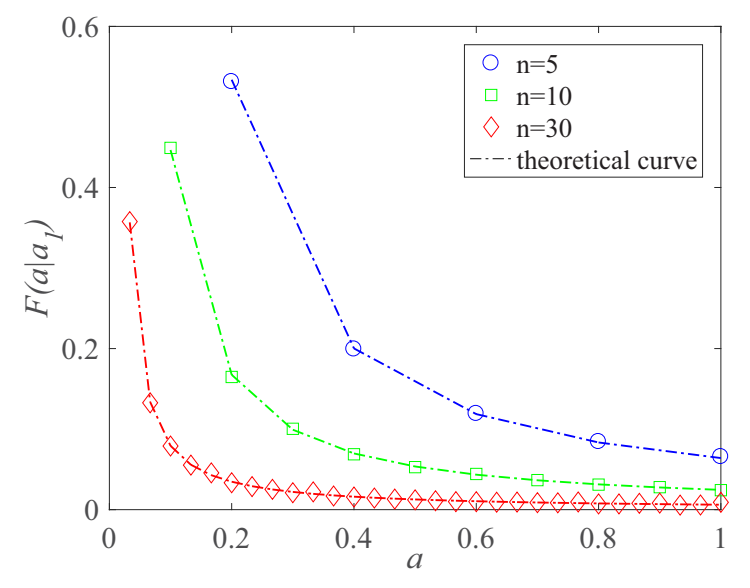

FIG. 1 . The probability of the nodes with activity $a_{1}$ connecting to the nodes with activity $a$ in the instantaneous network $G_{t}$, obtained from numerical simulation and theoretical analysis. We assume there are $n$ different activity classes, in which the nodes' activities are uniformly sampled in $(0,1]$ with the sampling interval $\frac{1}{n}$ and the activity distribution takes the form $F(a) \propto a^{-2}$. Here we choose $a_{1}$ as the minimum activity in the network. For the example of $n=5$, the activity is selected from $a \in\{0.2,0.4,0.6,0.8,1\}$ with $a_{1}=0.2$. The symbols (circle for $n=5$, square for $n=10$, diamond for $n=$ 30) and dotted lines represent the results of numerical simulations and theoretical analysis, respectively. The size of network is set to be $N=10000$ in each realization, and the simulation results are averaged over 100 realizations.

Since $G_{t}$ is undirected, $F\left(a, a^{\prime}\right)$ is symmetric with $F\left(a, a^{\prime}\right)=$ $F\left(a^{\prime}, a\right), \forall a, a^{\prime}$.

We define $F\left(a^{\prime} \mid a\right)$ as the conditional probability that an edge of nodes with activity $a$ points to a node with activity $a^{\prime}$. Then following the definition of degree correlation in static networks, $G_{t}$ is said to be uncorrelated in terms of activity if $F\left(a^{\prime} \mid a\right)=F_{n}\left(a^{\prime}\right)$ is satisfied for all $a$ and $a^{\prime}$.

From Eqs. (5) and (6), we derive the expression of $F\left(a^{\prime} \mid a\right)$ as

$$
F\left(a^{\prime} \mid a\right)=\frac{F\left(a^{\prime}, a\right)}{F_{n}(a)}=\frac{\frac{\left(a^{\prime}+a\right) F\left(a^{\prime}\right) F(a)}{2\langle a\rangle}}{\frac{(a+\langle a\rangle) F(a)}{2\langle a\rangle}}=\frac{\left(a^{\prime}+a\right) F\left(a^{\prime}\right)}{a+\langle a\rangle},
$$

which indicates the correlation in terms of activity in the instantaneous network $G_{t}$. Following the definition of normalized correlation function in Ref. [37], it can be concluded that at each time step $t$, the instantaneous network $G_{t}$ is disassortative mixing in terms of the node's activity.

In Fig. 1, we depict the probability distribution $F\left(a \mid a_{1}\right)$ of the nodes with activity $a_{1}$ connecting to the nodes with activity $a$ in the instantaneous network $G_{t}$. It is observed that the simulation results consist with the theoretical analysis.

\section{B. SAIS epidemic model}

To capture the risk perception and awareness diffusion on the network, we introduce the susceptible-alert-infectedsusceptible (SAIS) epidemic model that is coupled with the classical SIS model by adding an "Alert" compartment to account for the preventive behavior [38]. In this model, each node can be one out of three possible states: susceptible, alert or infectious. Both susceptible and alert nodes in contact with an infectious node can potentially be infected at a given constant infection rate, while the infection rate for the alert nodes is lower as a consequence of the preventive behavior. We denote the infection rate of susceptible nodes and alert nodes as $\lambda$ and $\tilde{\lambda}$, respectively. Also, each infected node will be recovered at another constant rate $\mu$.

As mentioned above, the threshold model is employed to describe the awareness diffusion and preventive behavior adoption on the network. The information for risk perception includes both the number of infected neighbors and that of alert neighbors. At each time step $t$, a susceptible node with activity $a$ will become alert once it has at least $c_{a}$ instantaneous infectious neighbors or at least $d_{a}$ alert neighbors in $G_{t}$. The two critical values $c_{a}$ and $d_{a}$ are defined as our infection threshold $\left(T_{i}\right)$ and alert threshold $\left(T_{a}\right)$, respectively. We assume that the alert state will be kept for $h$ time steps, depending on the duration of preventive effect.

Next, we describe the epidemic spreading process on the ADNs. Denote the fractions of susceptible, alert and infectious nodes in the class of activity $a$ as $S_{a}^{t}, A_{a}^{t}$, and $I_{a}^{t}$, respectively, then we have $S_{a}^{t}+A_{a}^{t}+I_{a}^{t}=1$ for each time step $t$. For any node $v$ with activity $a$ in the instantaneous network $G_{t}$, the probability of $v$ with degree $k$ connecting to $i$ infected nodes and $j$ alert nodes can be written as

$$
\begin{aligned}
\xi_{a}^{t}(k, i, j)= & P_{a}(k)\left(\begin{array}{c}
k \\
i
\end{array}\right)\left(\begin{array}{c}
k-i \\
j
\end{array}\right) \\
& \times\left(\Theta_{I, a}^{t}\right)^{i}\left(\Theta_{A, a}^{t}\right)^{j}\left(\Theta_{S, a}^{t}\right)^{k-i-j}
\end{aligned}
$$

with $\Theta_{I, a}^{t}=\sum_{a^{\prime}} F\left(a^{\prime} \mid a\right) I_{a^{\prime}}^{t}$ denoting the probability that an edge of nodes with activity $a$ points to an infected node in $G_{t}$. The definitions of $\Theta_{S, a}^{t}$ and $\Theta_{A, a}^{t}$ are similar to $\Theta_{I, a}^{t}$.

At time step $t$, each susceptible node will assess the local epidemic situation and make decision on whether it should adopt the preventive behavior. A susceptible node $v$ is alert at next time step if it is not infected and is aware of the epidemic spreading from risk perception. Then for node $v$ with degree $k$ that connects to $i$ infected neighbors and $j$ alert neighbors, the probability that $v$ is not infected and meets infection threshold condition $\left(i \geqslant c_{a}\right)$ can be written as

$$
\varphi_{1, a}^{S, t}=\sum_{k=c_{a}}^{k_{\max }} \sum_{i=c_{a}}^{k} \sum_{j=0}^{k-i} \xi_{a}^{t}(k, i, j)(1-\lambda)^{i} .
$$

Similarly, the probability of node $v$ staying healthy with alert threshold condition satisfied $\left(j \geqslant d_{a}\right)$ is

$$
\varphi_{2, a}^{S, t}=\sum_{k=d_{a}}^{k_{\max }} \sum_{j=d_{a}}^{k} \sum_{i=0}^{k-j} \xi_{a}^{t}(k, i, j)(1-\lambda)^{i},
$$

and the probability of node $v$ staying healthy with both the infection and alert threshold condition satisfied $\left(i \geqslant c_{a}, j \geqslant\right.$ $d_{a}$ ) is given by

$$
\varphi_{3, a}^{S, t}=\sum_{k=c_{a}+d_{a}}^{k_{\max }} \sum_{i=c_{a}}^{k-d_{a}} \sum_{j=d_{a}}^{k-i} \xi_{a}^{t}(k, i, j)(1-\lambda)^{i} .
$$


Then from the addition rule of probability, we can obtain the probability that a susceptible node $v$ with activity $a$ enters into alert state at next time step $t+1$

$$
\varphi_{a}^{S, t}=\varphi_{1, a}^{S, t}+\varphi_{2, a}^{S, t}-\varphi_{3, a}^{S, t} .
$$

Let $d_{a}=k_{\max }+1$ for all classes, then we have $\varphi_{a}^{S, t}=$ $\varphi_{1, a}^{S, t}$ and the risk perception is only related to the infectious neighbors, which has been considered in the Ref. [24].

Since the alert state can be kept only for $h$ time steps, an alert node will reassess the local epidemic situation for determining whether to adopt the preventive behavior again. For simplicity, we assume the decision-making threshold for alert nodes is the same as that for susceptible nodes. Then for an alert node $u$ with activity $a$ and entering into alert state at time step $t-h$, the probability $\varphi_{a}^{A, t}$ of $u$ being alert at time step $t+1$ can be simply derived by replacing the infection rate $\lambda$ in $\varphi_{a}^{S, t}$ with $\tilde{\lambda}$.

Define $\eta_{a}^{t}$ as the probability that a susceptible node with activity $a$ is not infected at time step $t$

$$
\eta_{a}^{t}=\sum_{k=0}^{k_{\max }} P_{a}(k)\left(1-\lambda \Theta_{I, a}^{t}\right)^{k},
$$

then replacing $\lambda$ with $\tilde{\lambda}$, we obtain $\tilde{\eta}_{a}^{t}$ as the probability of an alert node $u$ avoiding infection at time step $t$. For each alert node in the class of activity $a$ and entering into the alert state at time step $t-h$ (denoted as $A_{a}^{t, r}$ later), it remains alert during the successive $h-1$ time steps with probability $\prod_{l=1}^{h-1} \tilde{\eta}_{a}^{t-l}$. At time step $t$, it will reassess the local epidemic situation and become susceptible at next time step if it is not infected but abandons the preventive behavior. Such a case occurs with probability $\tilde{\eta}_{a}^{t}-\varphi_{a}^{A, t}$.

Let us denote $\phi_{a}^{t-m}, \gamma_{a}^{t-m}$ as

$$
\begin{aligned}
\phi_{a}^{t-m} & = \begin{cases}\tilde{\eta}_{a}^{t-m} & m>0 \\
\tilde{\eta}_{a}^{t}-\varphi_{a}^{A, t} & m=0,\end{cases} \\
\gamma_{a}^{t-m} & = \begin{cases}\tilde{\eta}_{a}^{t-m} & m \neq k h, k=0,1,2, \cdots \\
\varphi_{a}^{A, t-m} & m=k h, k=0,1,2, \cdots,\end{cases}
\end{aligned}
$$

the time evolution of three states in the class of activity $a$ can be written as

$$
\begin{gathered}
S_{a}^{t+1}=\mu I_{a}^{t}+S_{a}^{t} \eta_{a}^{t}-S_{a}^{t} \varphi_{a}^{S, t}+A_{a}^{t, r} \Phi_{a}^{t}, \\
A_{a}^{t+1}=S_{a}^{t} \varphi_{a}^{S, t}+A_{a}^{t} \tilde{\eta}_{a}^{t}-A_{a}^{t, r} \Phi_{a}^{t}, \\
I_{a}^{t+1}=1-\mu I_{a}^{t}-S_{a}^{t} \eta_{a}^{t}-A_{a}^{t} \tilde{\eta}_{a}^{t},
\end{gathered}
$$

with $\Phi_{a}^{t}=\prod_{l=1}^{h} \phi_{a}^{t+1-l}, \quad A_{a}^{t, r}=S_{a}^{t-h} \varphi_{a}^{S, t-h}+\sum_{i=2}^{\lfloor t / h\rfloor} S_{a}^{t-i h}$ $\varphi_{a}^{S, t-i h} \prod_{j=1}^{i-1}\left(\prod_{l=1}^{h} \gamma_{a}^{t+1-j h-l}\right)$.

The SAIS model for epidemic spreading process on the ADNs is obtained by extending the Eqs. (16)-(18) to each activity class. The epidemic threshold described as the ratio of infection rate to the recovery rate is a crucial parameter for characterizing the disease contagion process. Beyond the epidemic threshold, the epidemic would persist in the network eventually. It is worth noting that the epidemic threshold of the SAIS model in Eqs. (16)-(18) can be hardly obtained due to the threshold model of awareness diffusion and the finite duration of being alert. Thus we consider some special cases of $c_{a}, d_{a}$, and $h$ in the next section while more complex cases will resort to the numerical simulations.

\section{ANALYSIS}

In this section, we consider two special cases of the duration $h=1$ and $h=\infty$. In the former case, the effect of preventive behavior is unsustainable or determined by the recent risk perception as in the existing works [22-24], while in the latter case, it is permanent. Particularly, these two cases are capable of depicting some typical human behavioral responses in the real world. For example, whether or not to wear masks often depends on the current risk perception, while seeking vaccination might endow the individual with permanent immunity.

Following the definition in Eqs. (14) and (15), we have $\Phi_{a}^{t}=\tilde{\eta}_{a}^{t}-\varphi_{a}^{A, t}$ and $A_{a}^{t, r}=A_{a}^{t}$ for $h=1$. Then the time evolution of $A_{a}^{t+1}$ and $S_{a}^{t+1}$ in the SAIS model (16)-(18) can be rewritten as

$$
\begin{gathered}
S_{a}^{t+1}=\mu I_{a}^{t}+S_{a}^{t}\left(\eta_{a}^{t}-\varphi_{a}^{S, t}\right)+A_{a}^{t}\left(\tilde{\eta}_{a}^{t}-\varphi_{a}^{A, t}\right), \\
A_{a}^{t+1}=S_{a}^{t} \varphi_{a}^{S, t}+A_{a}^{t} \varphi_{a}^{A, t} .
\end{gathered}
$$

In the case of $h=\infty, \Phi_{a}^{t}=0$ is obtained, which then gives the expressions of $A_{a}^{t+1}$ and $S_{a}^{t+1}$ as

$$
\begin{gathered}
S_{a}^{t+1}=\mu I_{a}^{t}+S_{a}^{t} \eta_{a}^{t}-S_{a}^{t} \varphi_{a}^{S, t}, \\
A_{a}^{t+1}=S_{a}^{t} \varphi_{a}^{S, t}+A_{a}^{t} \tilde{\eta}_{a}^{t} .
\end{gathered}
$$

Based on the two special values of $h$, we inspect some cases with different information for risk perception. They are $h=1$ based on the number of infectious neighbors, and $h=\infty$ with or without consideration of the number of alert neighbors.

\section{A. Case of $h=1$}

For the case of $h=1$, we consider the risk perception only related to the infectious neighbors and thus $\varphi_{a}^{S, t}=\varphi_{a, 1}^{S, t}$ is obtained. First, we discuss the case of $c_{a}=1$, which means a susceptible node can either be infected or be alert once contacting with an infected neighbor. Under $c_{a}=1$, we can obtain

$$
\begin{aligned}
\varphi_{a}^{S, t} & =\sum_{k=1}^{k_{\max }} \sum_{i=1}^{k} P_{a}(k)\left(\begin{array}{l}
k \\
i
\end{array}\right)\left(\Theta_{I, a}^{t}\right)^{i}\left(1-\Theta_{I, a}^{t}\right)^{k-i}(1-\lambda)^{i} \\
& =\sum_{k=1}^{k_{\max }} P_{a}(k) \sum_{i=1}^{k}\left(\begin{array}{l}
k \\
i
\end{array}\right)\left[(1-\lambda) \Theta_{I, a}^{t}\right]^{i}\left(1-\Theta_{I, a}^{t}\right)^{k-i} \\
& =\sum_{k=1}^{k_{\max }} P_{a}(k)\left[\left(1-\lambda \Theta_{I, a}^{t}\right)^{k}-\left(1-\Theta_{I, a}^{t}\right)^{k}\right] .
\end{aligned}
$$

Near the epidemic threshold, the probability of nodes being infected can be assumed to be $I_{a}^{t}=\epsilon \ll 1$, which gives the following approximations: $\left(1-\lambda \Theta_{I, a}^{t}\right)^{k} \approx 1-k \lambda \Theta_{I, a}^{t},(1-$ $\left.\Theta_{I, a}^{t}\right)^{k} \approx 1-k \Theta_{I, a}^{t}$. Then from Eq. (7), Eq. (23) can be 
rewritten as

$$
\begin{aligned}
\varphi_{a}^{S, t} & =\sum_{k=1}^{k_{\max }} k P_{a}(k)(1-\lambda) \Theta_{I, a}^{t} \\
& =(1-\lambda) m(a+\langle a\rangle) \sum_{a^{\prime}} \frac{\left(a+a^{\prime}\right) F\left(a^{\prime}\right)}{a+\langle a\rangle} I_{a^{\prime}}^{t} \\
& =(1-\lambda) m \sum_{a^{\prime}}\left(a+a^{\prime}\right) F\left(a^{\prime}\right) I_{a^{\prime}}^{t} \\
& =(1-\lambda) m\left(a I^{t}+\theta^{t}\right)
\end{aligned}
$$

with $I^{t}=\sum_{a^{\prime}} F\left(a^{\prime}\right) I_{a^{\prime}}^{t}, \theta^{t}=\sum_{a^{\prime}} a^{\prime} F\left(a^{\prime}\right) I_{a^{\prime}}^{t}$. Also, the probability $\eta_{a}^{t}$ in Eq. (13) is given by

$$
\begin{aligned}
\eta_{a}^{t} & =\sum_{k=0}^{k_{\max }} P_{a}(k)\left(1-\lambda \Theta_{I, a}^{t}\right)^{k} \\
& \approx \sum_{k=0}^{k_{\max }} P_{a}(k)\left(1-k \lambda \Theta_{I, a}^{t}\right)=1-\lambda m\left(a I^{t}+\theta^{t}\right) .
\end{aligned}
$$

With Eqs. (19), (20), (24), and (25), the SAIS epidemic model (16)-(18) is simplified as

$$
\begin{gathered}
S_{a}^{t+1}=\mu I_{a}^{t}-\left(S_{a}^{t}+A_{a}^{t}\right)\left(1-m a I^{t}-m \theta^{t}\right), \\
A_{a}^{t+1}=\left[(1-\lambda) S_{a}^{t}+(1-\tilde{\lambda}) A_{a}^{t}\right]\left(m a I^{t}+m \theta^{t}\right), \\
I_{a}^{t+1}=(1-\mu) I_{a}^{t}+\left(\lambda S_{a}^{t}+\tilde{\lambda} A_{a}^{t}\right)\left(m a I^{t}+m \theta^{t}\right) .
\end{gathered}
$$

This model can be viewed as an extension of the paradigmatic SIS epidemic model on ADNs proposed in Ref. [6].

Since the risk perception is only based on the infectious neighbors, the number of alert nodes is positively related to that of the infected nodes from the expression of $A_{a}^{t+1}$ in Eq. (27). Thus we have $A_{a}^{t+1}=\varepsilon I_{a}^{t+1}=\varepsilon \epsilon \ll 1$ with some constant $\varepsilon>0$. Leveraging the linear approximation in $I_{a}^{t}$ and $A_{a}^{t}$ that avoids the connections between two infected nodes or between an alert node and an infected one [6], the expression of $I_{a}^{t+1}$ in Eq. (28) can be rewritten as

$$
\begin{aligned}
I_{a}^{t+1} & =(1-\mu) I_{a}^{t}+\lambda S_{a}^{t}\left(m a I^{t}+m \theta^{t}\right) \\
& \approx(1-\mu) I_{a}^{t}+\lambda\left(m a I^{t}+m \theta^{t}\right) .
\end{aligned}
$$

This equation is exactly the same as the time evolution of the number of infectious nodes with activity $a$ in the SIS model [6], which does not consider awareness diffusion. Thus the epidemic threshold will remain unchanged, which can be expressed as

$$
\frac{\lambda}{\mu}>\lambda_{c}=\frac{1}{m\left(\langle a\rangle+\sqrt{\left\langle a^{2}\right\rangle}\right)} .
$$

Since the probability of a susceptible node being alert decreases with more infectious neighbors requested in the preventive behavior adoption, we can conclude that the epidemic threshold is not changed for the case of $c_{a}>1$. Thus when the effect of preventive behavior is not sustainable, the risk perception grounded on the number of infectious neighbors would not affect the epidemic threshold. This result is the same as that in Ref. [24] in which the node's awareness is updated with the ratio of previous contacts with infectious nodes on a training window.

\section{B. Case of $\boldsymbol{h}=\infty$}

For the case of $h=\infty$, the impact of different information for risk perception on the epidemic threshold will be discussed. First, we consider that the risk perception is only based on the number of infectious neighbors so that $d_{a}=\infty$ is assumed. For the case of $c_{a}=1$ where a susceptible node might be alert contacting with an infectious neighbors, from the Eqs. (21), (22), (24), and (25), we obtain the SAIS model

$$
\begin{gathered}
S_{a}^{t+1}=S_{a}^{t}+\mu I_{a}^{t}-S_{a}^{t}\left(m a I^{t}+m \theta^{t}\right), \\
A_{a}^{t+1}=A_{a}^{t}+\left[(1-\lambda) S_{a}^{t}-\tilde{\lambda} A_{a}^{t}\right]\left(m a I^{t}+m \theta^{t}\right), \\
I_{a}^{t+1}=(1-\mu) I_{a}^{t}+\left(\lambda S_{a}^{t}+\tilde{\lambda} A_{a}^{t}\right)\left(m a I^{t}+m \theta^{t}\right) .
\end{gathered}
$$

In the steady state for $t \rightarrow \infty, S_{a}^{t+1}=S_{a}^{t}, I_{a}^{t+1}=I_{a}^{t}$, and $A_{a}^{t+1}=A_{a}^{t}$ are derived. Under the model (31)-(33), we have

$$
\begin{aligned}
\mu I_{a}^{t}-S_{a}^{t}\left(m a I^{t}+m \theta^{t}\right) & =0, \\
(1-\lambda) S_{a}^{t}-\tilde{\lambda} A_{a}^{t} & =0,
\end{aligned}
$$

which gives $S_{a}^{t}=\frac{\mu I_{a}^{t}}{m a I^{t}+m \theta^{t}}, A_{a}^{t}=\frac{(1-\lambda) S_{a}^{t}}{\tilde{\lambda}}$. With $S_{a}^{t}+I_{a}^{t}+$ $A_{a}^{t}=1$, we obtain

$$
I_{a}^{t}=\frac{m a I^{t}+m \theta^{t}}{\frac{\mu}{\tilde{\lambda}}(1-\lambda+\tilde{\lambda})+m a I^{t}+m \theta^{t}} .
$$

Introducing Eq. (34) into $I^{t}=\sum_{a} F(a) I_{a}^{t}, \quad \theta^{t}=$ $\sum_{a} a F(a) I_{a}^{t}$, we have the following two self-consistency equations

$$
\begin{aligned}
I^{t} & =\sum_{a} F(a) \frac{m a I^{t}+m \theta^{t}}{\frac{\mu}{\tilde{\lambda}}(1-\lambda+\tilde{\lambda})+m a I^{t}+m \theta^{t}}, \\
\theta^{t} & =\sum_{a} a F(a) \frac{m a I^{t}+m \theta^{t}}{\frac{\mu}{\tilde{\lambda}}(1-\lambda+\tilde{\lambda})+m a I^{t}+m \theta^{t}} .
\end{aligned}
$$

In addition, we define $F\left(I^{t}, \theta^{t}\right)=\sum_{a} F(a) I_{a}^{t}-I^{t}$, $G\left(I^{t}, \theta^{t}\right)=\sum_{a} a F(a) I_{a}^{t}-\theta^{t}$. Let $I_{a}^{t}=0 \forall a$, we have $I^{t}=$ $0, \theta^{t}=0$, and $F(0,0)=0, G(0,0)=0$ by Eqs. (35) and (36). Let $I_{a}^{t}=1 \quad \forall a$, we have $I^{t}=1, \quad \theta^{t}=\langle a\rangle$ and $F(1,\langle a\rangle)<0, G(1,\langle a\rangle)<0$. Then the equation set $F\left(I^{t}, \theta^{t}\right)=0, G\left(I^{t}, \theta^{t}\right)=0$ has a nontrivial solution if the system is unstable in the disease-free state. Expanding the $F$ and $G$ at second order in $I^{t}$ and $\theta^{t}$, we obtain the Jacobian matrix

$$
\left[\begin{array}{cc}
\frac{m\langle a\rangle}{\mu}-1 & \frac{m}{\bar{\mu}(1-\lambda+\tilde{\lambda})}-\frac{\mu}{\bar{\lambda}(1-\lambda+\tilde{\lambda})} \\
\frac{m\left\langle a^{2}\right\rangle}{\frac{\mu}{\lambda}(1-\lambda+\tilde{\lambda})} & \frac{m\langle a\rangle}{\frac{\mu}{\lambda}(1-\lambda+\tilde{\lambda})}-1
\end{array}\right] .
$$

To obtain the epidemic threshold for the system, the largest eigenvalues of (37) are required to be larger than 0 . That is,

$$
\Lambda_{\max }=\frac{m\langle a\rangle}{\frac{\mu}{\tilde{\lambda}}(1-\lambda+\tilde{\lambda})}-1+\frac{m \sqrt{\left\langle a^{2}\right\rangle}}{\frac{\mu}{\tilde{\lambda}}(1-\lambda+\tilde{\lambda})}>0,
$$


which delivers the condition for the presence of an endemic state

$$
\frac{\lambda}{\mu}>\frac{\lambda(1-\lambda+\tilde{\lambda})}{\tilde{\lambda}} \frac{1}{m\left(\langle a\rangle+\sqrt{\left\langle a^{2}\right\rangle}\right)} .
$$

If $\tilde{\lambda}$ is proportional to $\lambda$, i.e., $\tilde{\lambda}=c \lambda$ with a constant $0<c \leqslant$ 1 , then the Eq. (38) can be rewritten as

$$
\frac{\lambda}{\mu}>\lambda_{c}=\frac{1}{\mu(1-c)+c m\left(\langle a\rangle+\sqrt{\left\langle a^{2}\right\rangle}\right)} .
$$

Since $\lambda \geqslant \tilde{\lambda}$, we have $\frac{\lambda(1-\lambda+\tilde{\lambda})}{\tilde{\lambda}}>1$ and compared with the Eq. (30), the epidemic threshold is enhanced. Thus, the longer duration of preventive effect is in a position to suppress the epidemic outbreak.

For the case of $c_{a}>1$ and near the epidemic threshold, the fraction of infected nodes can be assumed to be $\epsilon \ll 1$ when $t \rightarrow \infty$. Then the events in which a susceptible node connects to at least $c_{a}$ infected nodes are not considered, i.e., $\varphi_{a, 1}^{S, t} \rightarrow 0$. Since $d_{a}=\infty$, we have $\varphi_{a}^{S, t}=\varphi_{a, 1}^{S, t} \rightarrow 0$. Introducing $\varphi_{a}^{S, t} \rightarrow 0$ into the Eq. (22), then in the steady state, $A_{a}^{t}$ could no longer be denoted by $S_{a}^{t}$ and $I_{a}^{t}$. Thus it is hard to conduct further analysis on this case by available analytical approaches. Nevertheless, if $c_{a}$ is too large to trigger the awareness diffusion, i.e., $\varphi_{a}^{S, t} \rightarrow 0 \forall t \geqslant 0$, the SAIS model turns into the typical SIS model and thus the epidemic threshold will keep unchanged.

Next we consider the risk perception based on both the number of infectious neighbors and that of alert neighbors. Since the threshold model adopted for awareness diffusion might trigger different types of awareness diffusion process, it is an intricate task to deal with the epidemic threshold problem for general cases of $c_{a}$ and $d_{a}$ with the SAIS model given in (21)-(22). The following analysis is proceeded under the assumption of $\varphi_{a}^{S, t}>0$ that implies the possibility of preventive behavior adoption when $t \rightarrow \infty$. This assumption could be held with a reasonable combination of the infection and alert thresholds, such as $c_{a}=1, d_{a}=1$. In the later analysis of numerical simulation, it is observed that under the given topological parameters of the ADN, this assumption could be applied to a number of other cases with more conservative threshold conditions. Here, we provide a general analysis for these cases in which the value of $\varphi_{a}^{S, t}$ is dependent on the $c_{a}$ and $d_{a}$. Under this assumption, the steady state of the SAIS model in (21)-(22) is satisfied with

$$
I_{a}^{t}=\frac{\tilde{\lambda}\left(m a I^{t}+m \theta^{I}\right)\left[\lambda\left(m a I^{t}+m \theta^{I}\right)+\varphi_{a}^{S, t}\right]}{\mu\left[\tilde{\lambda}\left(m a I^{t}+m \theta^{I}\right)+\varphi_{a}^{S, t}\right]+\tilde{\lambda}\left(m a I^{t}+m \theta^{I}\right)\left[\lambda\left(m a I^{t}+m \theta^{I}\right)+\varphi_{a}^{S, t}\right]},
$$

when $t \rightarrow \infty$. Similarly to the analysis of Eq. (34), the Jacobian matrix of this system can be derived as

$$
\left[\begin{array}{lc}
\frac{\tilde{\lambda} m\langle a\rangle}{\mu}-1 & \frac{\tilde{\lambda} m}{\mu} \\
\frac{\tilde{\lambda} m\left\langle a^{2}\right\rangle}{\mu} & \frac{\tilde{\lambda} m\langle a\rangle}{\mu}-1
\end{array}\right]
$$

whose largest eigenvalue is given by $\Lambda_{\max }=\frac{\tilde{\lambda} m}{\mu}(\langle a\rangle+$ $\left.\left\langle a^{2}\right\rangle\right)-1$. Then we obtain

$$
\frac{\tilde{\lambda}}{\mu}>\frac{1}{m\left(\langle a\rangle+\left\langle a^{2}\right\rangle\right)} .
$$

Equation (41) shows that the condition for the presence of an endemic state is dependent on the infection rate $\tilde{\lambda}$ of alert nodes while unrelated to the $\lambda$ of susceptible nodes. This result is understandable since the susceptible nodes all become alert with awareness diffusion and a permanent preventive effect under the assumption of $\varphi_{a}^{S, t}>0$. Obviously, Eq. (41) provides the upper bound of the epidemic threshold under the SAIS model (16)-(18).

\section{NUMERICAL SIMULATIONS}

Based on the proposed SAIS model, we perform extensive numerical simulations to corroborate the theoretical results and further investigate the role of awareness diffusion in the epidemic spreading process. Under the observation of heavytailed activity distributions in empirical networks $[6,39]$, here we take $F(a) \propto a^{-2}$. For simplicity, we restrict $a$ selected from five candidates, i.e., $a \in\{0.5,0.6,0.7,0.8,0.9\}$, and the number of edges created by an active node is chosen to be $m=6$. In the following simulations, the size of network, the recovery rate and the initial proportion of infectious nodes chosen randomly in the population are set to be $N=5000$, $\mu=1$, and $I^{0}=0.1$, respectively. If not specified, we assume $\tilde{\lambda}=c \lambda$ with $c=0.5$. The theoretical results of $S_{a}^{t}, A_{a}^{t}$, and $I_{a}^{t}$ in the class of activity $a$ are derived following the Eqs. (8)(18). All the Monte Carlo simulation results are obtained by averaging the results over 20 independent realizations.

Firstly, to check the validness of our statistical model in characterizing the epidemic spreading process on the ADNs, we depict the time evolution of $S^{t}, A^{t}, I^{t}$ according to the SAIS model in Eqs. (16)-(18) and Monte Carlo simulations under different cases of risk perception. The risk perception only based on the number of infectious neighbors and that also incorporating the number of alert neighbors are considered. For each case, both the endemic and disease-free states of the propagation system are studied. The results are shown in Fig. 2. Under these two cases, the simulation results are observed to match well with the theoretical results.

Next, we consider the impact of risk perception only based on the number of infectious neighbors and thus $T_{a}=\infty$ is given. In Fig. 3, we depict the growth of the final fraction of infected nodes $I^{\infty}$ versus the infection rate $\lambda$ under different $T_{i}$ s and durations $h$. With the given parameters $m, a$, and $F(a)$ of the ADN, the epidemic threshold without risk perception is calculated to be $\lambda_{c}=\frac{1}{m\left(\langle a\rangle+\sqrt{\left\langle a^{2}\right\rangle}\right)}=0.1284$. According to the analysis in Sec. III A, the epidemic threshold stays unchanged when the preventive effect is valid only for one time step. From Fig. 3(a), the epidemic thresholds suggested by Monte Carlo simulations and the SAIS model are close to $\lambda_{c}$, and 

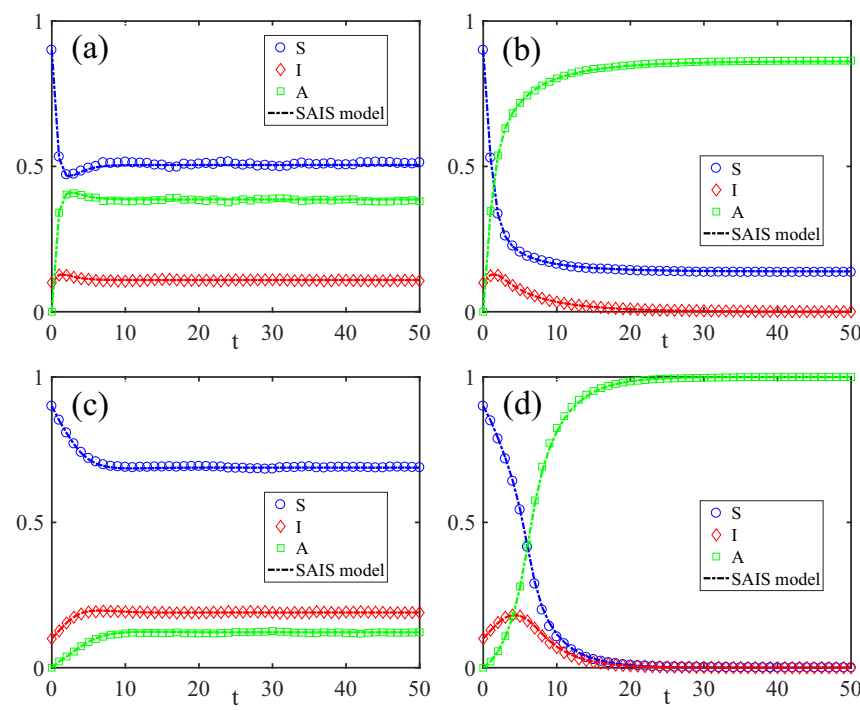

FIG. 2. Time evolution of $S^{t}, A^{t}$ and $I^{t}$ under different cases of risk perception with the infection rate $\lambda=0.2$. The results are obtained when (a) $T_{i}=1, T_{a}=\infty, h=1$; (b) $T_{i}=1, T_{a}=\infty, h=$ $\infty$; (c) $T_{i}=3, T_{a}=3, h=1$; (d) $T_{i}=3, T_{a}=3, h=\infty$. The symbols and dotted lines represent the results of Monte Carlo simulation and the SAIS model, respectively. Other parameters are set to be $N=5000, I^{0}=0.1, c=0.5, \mu=1$.

thus the analytical results in Sec. III A are corroborated. However, although the epidemic threshold is not affected by the preventive behavior adoption, the infection prevalence is reduced as shown in Fig. 3(a).

For the case of permanent preventive effect in Fig. 3(b), it is observed that in addition to the reduction of infection prevalence, the epidemic threshold increases significantly. To further explore the impact of awareness diffusion on the epidemic threshold under the risk perception with $T_{i}=1, T_{a}=$ $\infty$, and $h=\infty$, we record the epidemic thresholds under a series of different alert infection rates $\tilde{\lambda}=c \lambda$. The results are presented in Fig. 4. It is obvious that a lower infection rate of alert nodes is beneficial to enhance the epidemic threshold. As shown in Fig. 4, the epidemic thresholds $\lambda_{c}$ obtained from
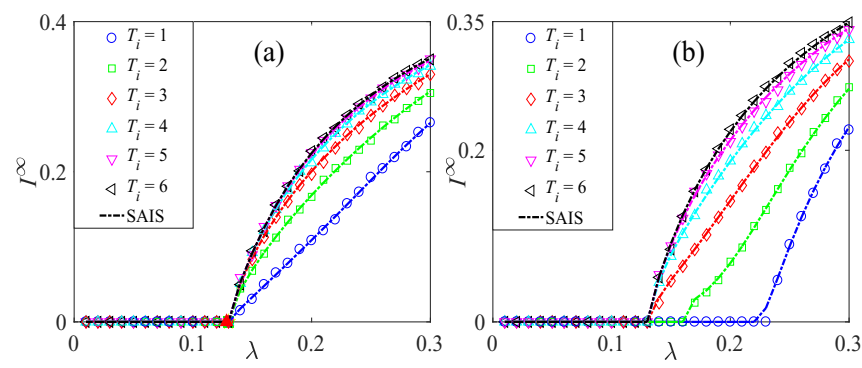

FIG. 3. Final fraction of infected nodes as a function of the infection rate $\lambda$, for different $T_{i}$ when the alert threshold is set to be $T_{a}=\infty$. In the figure, (a) $h=1$ and (b) $h=\infty$. The epidemic threshold suggested by Monte Carlo simulations and the SAIS model are recorded as soon as $I^{\infty}$ is slightly larger than 0.015 (the critical value above which the realization is considered as endemic). In (a), the epidemic threshold given by theoretical analysis is labeled by red triangle. Other parameters are set to be the same as those in Fig. 2.

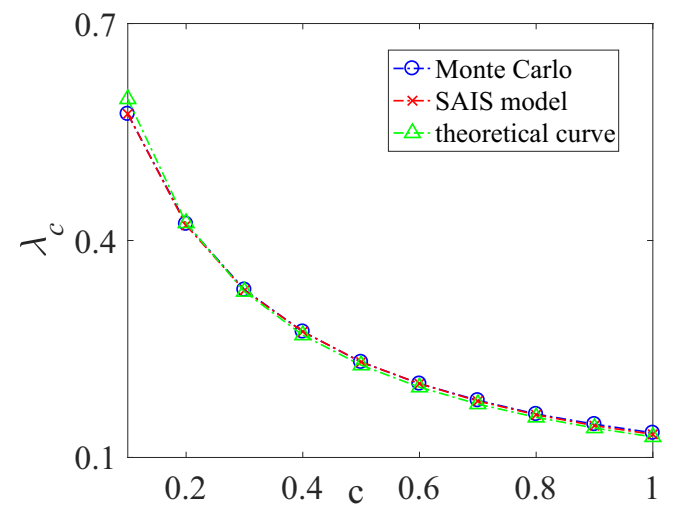

FIG. 4. Epidemic threshold under different infection rates of alert nodes $\tilde{\lambda}=c \lambda$. The epidemic thresholds suggested by Monte Carlo simulations and the SAIS model (31)-(33) are recorded following the same steps in Fig. 3. Other parameters are set to be the same as those in Fig. 2.

Monte Carlo simulations and the SAIS model are approximate to those given by the Eq. (39).

In Fig. 5, we investigate the impact of incorporating the number of alert neighbors in risk perception. We plot the $I^{\infty}$ under different threshold conditions $\left(T_{i}\right.$ and $\left.T_{a}\right)$ versus infection rate $\lambda$, when the duration is set to be $h=1$. According to the results in Figs. 3(a) and 5(a), the epidemic threshold is enhanced by including the information of the number of alert neighbors. Also, the infection prevalence is reduced. While the infectious neighbors are necessary for starting the process of awareness diffusion, the results in Fig. 5 demonstrate that the information of alert neighbors is important for raising the epidemic threshold and reducing infection prevalence. Furthermore, there are also some interesting phenomena revealed by our model. In Fig. 5(d) with $T_{i}=4$ and $T_{a}=2$, we find that the final fraction of infected nodes $I^{\infty}$ has an abrupt jump from 0.144 to 0 when the infection rate is set to be $\lambda \approx 0.17$. Specifically, $I^{\infty}$ increases with the increasing $\lambda$ from 0.13 to 0.17 , and suddenly jumps to 0 at $\lambda \approx 0.17$. Then it remains 0 until $\lambda \approx 0.25$, after which it raises gradually again with an increasing $\lambda$. In order to further investigate the first-order transition of $I^{\infty}$ here, we perform large amounts of simulations for pinpointing the spreading process near $\lambda=0.17$.

In Fig. 6, we depict the time evolution of the fractions of infected and alert nodes, as well as the final values of them for the infection rate varying in the interval $[0.11,0.3]$ with a discrete step of 0.001 . From Figs. 6(a) and 6(c), it can be seen that the abrupt transition (from a positive value to 0 ) of $I^{\infty}$ is exactly accompanied by the abrupt jumping of $A^{\infty}$ from 0 to a positive value close to 1 . The infection rate where the transition occurs is defined as our critical infection rate $\lambda^{*}$. Then it is observed that when $\lambda<\lambda^{*}$, the final fraction of alert nodes $A^{\infty}$ is approximate to 0 , which means that the awareness diffusion is limited. Consequently, $I^{\infty}$ with $T_{a}=2$ is almost the same to that with a larger $T_{a}$ as shown in Fig. 5(d). For $\lambda$ close to but greater than $\lambda^{*}, A^{\infty}$ is approximate to 0.9583 and thus the cascade of awareness on the network could be expected. Since the alert nodes have a reduced infection risk by taking self-protecting measures, the epidemic spreading process is suppressed and 

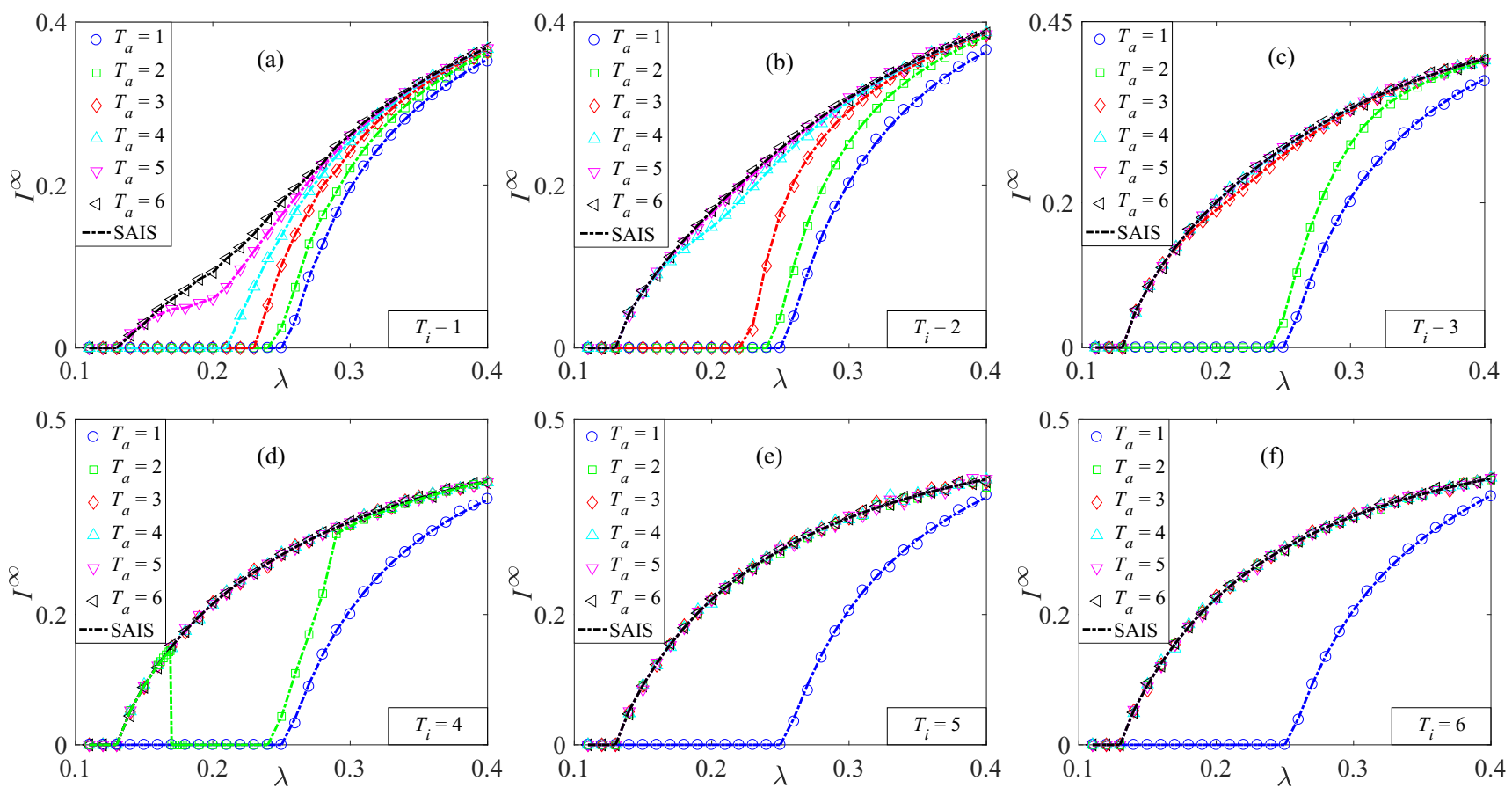

FIG. 5. Final fraction of infected nodes $I^{\infty}$ as a function of infection rate $\lambda$, for different $T_{i} \mathrm{~s}$ and $T_{a} \mathrm{~s}$ with duration $h=1$. From (a) to (f), $T_{i}$ is respectively fixed at $1,2,3,4,5,6$, and $T_{a}=\{1,2,3,4,5,6\}$. The epidemic thresholds suggested by Monte Carlo simulations and the SAIS model are recorded following the same steps in Fig. 3. Other parameters are set to be the same as those in Fig. 2.

finally disappears on the network. Such cascade effects are dominated in the coupled dynamical process of infection and awareness for a larger $\lambda$ until the condition in the Eq. (41) is met, where the disease contagion breaks out in spite of the preventive measures. In Fig. 6(c), $A^{\infty}$ decays continuously from $\lambda=0.25$ that is close to the epidemic threshold given by
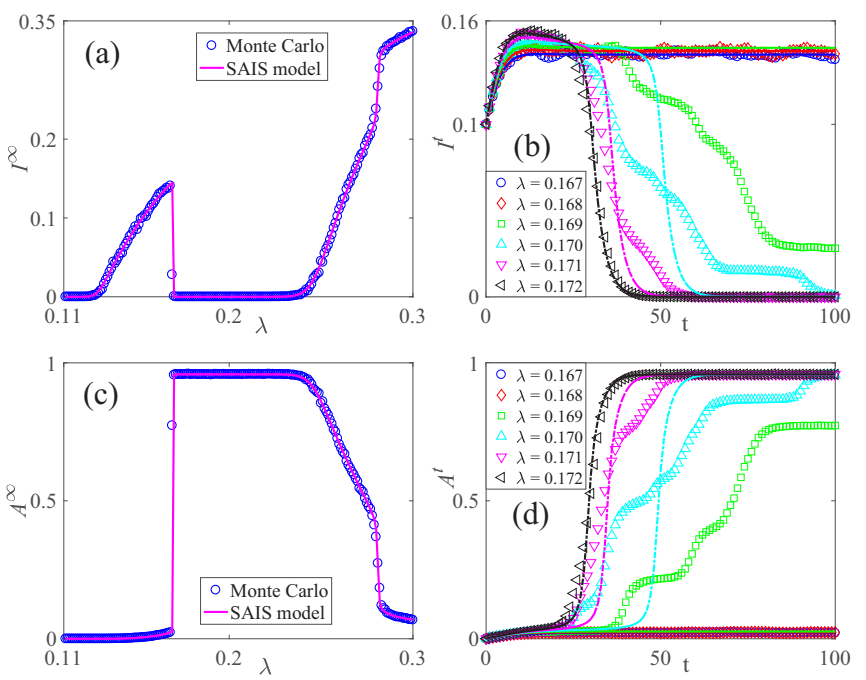

FIG. 6. Coupled dynamics of infection and awareness for the risk perception with $h=1, T_{i}=4, T_{a}=2$. In the figure, (a) final fraction of infected nodes $I^{\infty}$, (b) time evolution of infected nodes $I^{t}$, (c) final fraction of alert nodes $A^{\infty}$, and (d) time evolution of alert nodes $A^{t}$. In (b) and (d), the symbols and dotted lines represent the results of Monte Carlo simulation and the SAIS model, respectively. Other parameters are set to be the same as those in Fig. 2. $\lambda(\tilde{\lambda}) / \mu=0.2568(0.1284)$. Then, $I^{\infty}$ increases continuously as shown in Fig. 6(a). It is of significant interest to understand the dynamical process of the propagation system near the transition, i.e., when $\lambda$ is very close to $\lambda^{*}$. In Figs. 6(b) and 6(d), we recored the time evolution of $I^{t}$ and $A^{t}$, respectively. It is observed that with a slight increase of $\lambda$ close to $\lambda^{*}, I^{t}$ increases gradually over a period of time from $t=0$, then it decreases to 0 with time. In the meanwhile, $A^{t}$ increases with the slightly increasing of $\lambda$ and then it arrives at a stable value with time. In addition, it can be seen that the decrease of $I^{t}$ and also the increase of $A^{t}$ are rather abrupt, and the time of this abrupt change is advanced with a larger $\lambda$. Note that during a period of time from $t=0$, both the $I^{t}$ and $A^{t}$ are increased with $\lambda$. According to the threshold model of awareness diffusion in our SAIS model, a certain proportion of infected nodes is the indispensable ingredient for triggering the cascade of awareness. Thus the results of Figs. 6(b) and 6(d) might provide an explanation for the abrupt first-order transition in the system: when $\lambda<\lambda^{*}$, the awareness diffusion is impeded and the outbreak of disease contagion occurs. For $\lambda>\lambda^{*}$, the larger infection prevalence in the initial period promotes the diffusion of awareness, which on the contrary, suppresses the epidemic spreading process instead. While $\lambda$ is large enough to surmount the effect of preventive measures, the disease contagion certainly breaks out.

Figure 7 provides the growth of $I^{\infty}$ under different threshold conditions $T_{i}$ and $T_{a}$ versus infection rate $\lambda$, when the duration is set to be $h=\infty$. From Figs. 5 and 7, both the epidemic threshold and infection prevalence are shifted by the permanent preventive effect. In Figs. 7(a) and 7(b), the final fraction of infected nodes with different alert thresholds are almost identical to each other under the given infection 

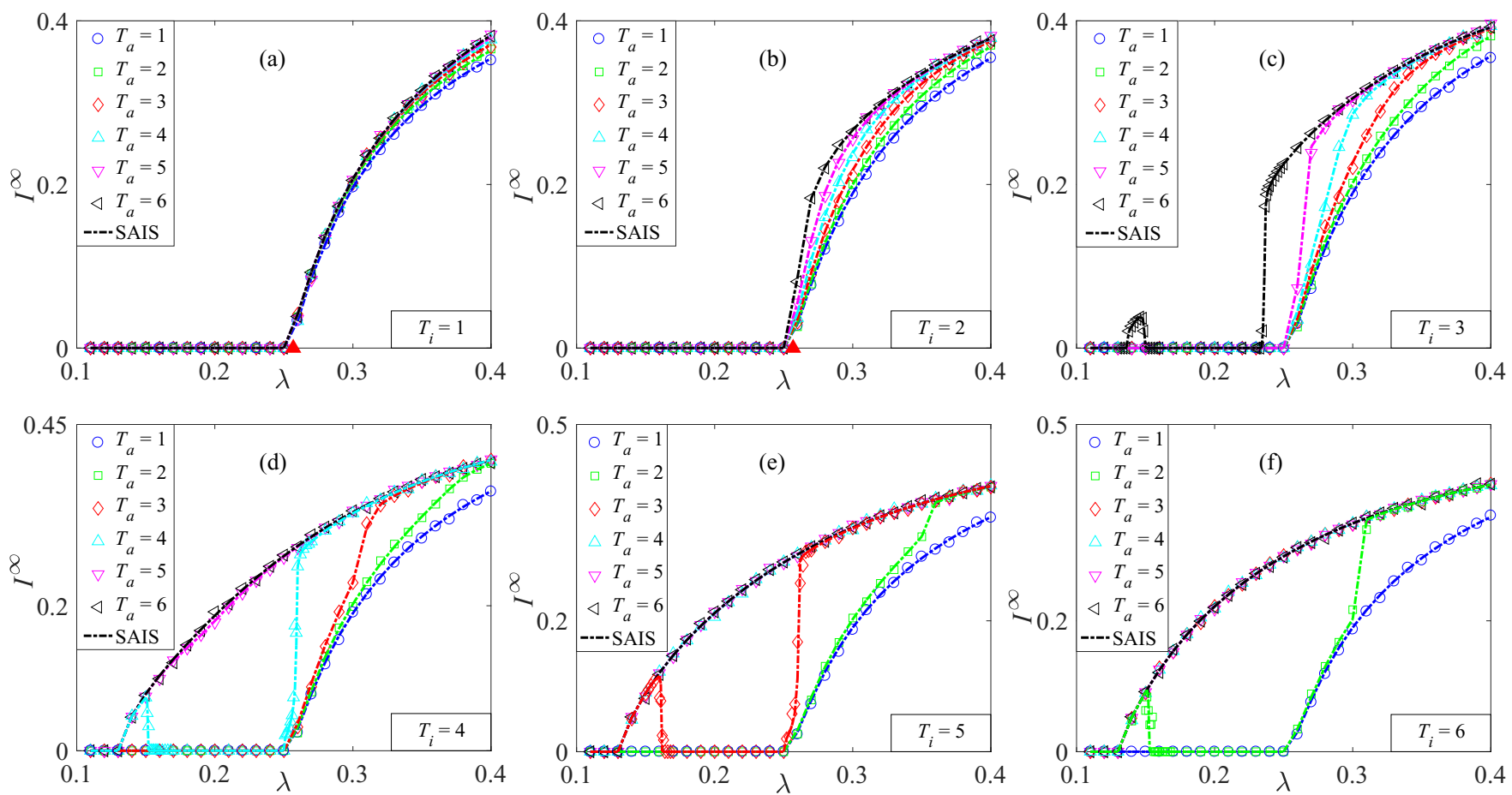

FIG. 7. Final fraction of infected nodes $I^{\infty}$ as a function of infection rate $\lambda$, for different $T_{i}$ s and $T_{a}$ s with duration $h=\infty$. From (a) to (f), $T_{i}$ is respectively fixed at $1,2,3,4,5,6$ and $T_{a}=\{1,2,3,4,5,6\}$. The epidemic thresholds suggested by Monte Carlo simulations, the SAIS model and theoretical analysis are recorded and labeled following the same steps in Fig. 3. Other parameters are set to be the same as those in Fig. 2.

rate. According to the theoretical analysis in Sec. III B, the epidemic threshold is only dependent on the infection rate $\tilde{\lambda}$ under the assumption $\varphi_{a}^{S}(t)>0$. As shown in Fig. 7, this assumption is held with $T_{i}=1,2$ for $T_{a}$ from 1 to 6 , and the epidemic thresholds suggested by the Monte Carlo simulations as well as the SAIS model are close to the theoretical one. In addition, the abrupt first-order transition of $I^{\infty}$ is also observed under a variety of threshold conditions in Figs. 7(c)7(f), such as $T_{i}=3, T_{a}=6$ in Fig. 7(c) and $T_{i}=4, T_{a}=4$ in Fig. 7(d). Similar analysis of the dynamical process near these transitions indicates that the effect of awareness diffusion with $h=\infty$ presents a similar two-stage characteristic as shown in Fig. 5. That is, there exists a critical infection rate $\lambda^{*}>$ $\frac{1}{m\left(\langle a\rangle+\sqrt{\left\langle a^{2}\right\rangle}\right)}$, below which the awareness diffusion is limited and the disease contagion outbreaks on the networks. On the contrary, a slight increase of $\lambda=\lambda^{*}$ will trigger the cascade of awareness and suppress the epidemic spreading instead. These cases are reminiscent of the real-world phenomena where a virus that may not be highly contagious would, instead, persist for a long time.

Furthermore, we investigate the effects of time-varying network structure affected by awareness diffusion on disease contagion. Under the framework of ADNs and inspired by the previous works [22,23], we introduce the time-varying activity of alert nodes in the coupled dynamical process of infection and awareness. The joint effect of preventive behavior adoption and activity reduction on suppressing the disease contagion will be studied. Specifically, we assume that the alert node with activity $a$ will reduce its activity to $\tilde{a}=\gamma a$ with $\gamma \leqslant 1$ until it leaves the alert state. Four candidates of $\gamma$ are employed in the simulations, which are $0.25,0.5,0.75$ and 1 . Since we have set $m=6$, we consider three cases of threshold conditions with $T_{i}+T_{a}=6$. The final fraction of infected nodes with time-varying activity is presented in Fig. 8. From Figs. 8(a) to 8(c) with duration $h=1$, we find a nontrivial interaction among the epidemic spreading, the awareness diffusion and the time-varying network structure. In Fig. 8(a), the final fraction of infected nodes $I^{\infty}$ with $\gamma=1$ is lower than other three candidates of $\gamma$ for $\lambda \in[0.17,0.25]$, suggesting that a reduced activity is not always beneficial to restrain epidemic spreading. This result is understandable in view of the significant role of alert nodes in awareness diffusion. Under the proposed SAIS model, the activity reduction of alert nodes delivers two opposite effects: it reduces the infection risk of alert nodes but also hampers the diffusion of awareness. When $h=1$, the effect of preventive measures is determined by the recent risk perception, and thus the alert nodes may fail to keep alert and thereby decelerate the awareness diffusion over the whole networks. In Fig. 8(b), $I^{\infty}$ seems not to be affected by the time-varying activity of alert nodes due to the localized awareness diffusion shown in Fig. 5(c). In Fig. 8(c), the curves of $I^{\infty}$ with $\gamma=0.25$ and 0.5 are obviously higher than that with $\gamma=1$, thus a reduced activity related to $\gamma=0.25$ and 0.5 would be detrimental for controlling the disease contagion. Nevertheless, $I^{\infty}$ with $\gamma=$ 0.75 is decreased compared with $\gamma=1$ for $\lambda<0.31$. Thus it can be concluded that there is an optimal $\gamma$ for balancing these two opposite effects. Figs. 8(d)-8(f) are related to $h=\infty$ where the effect of preventive behavior is permanent. For the three cases of threshold conditions, it is observed that com- 

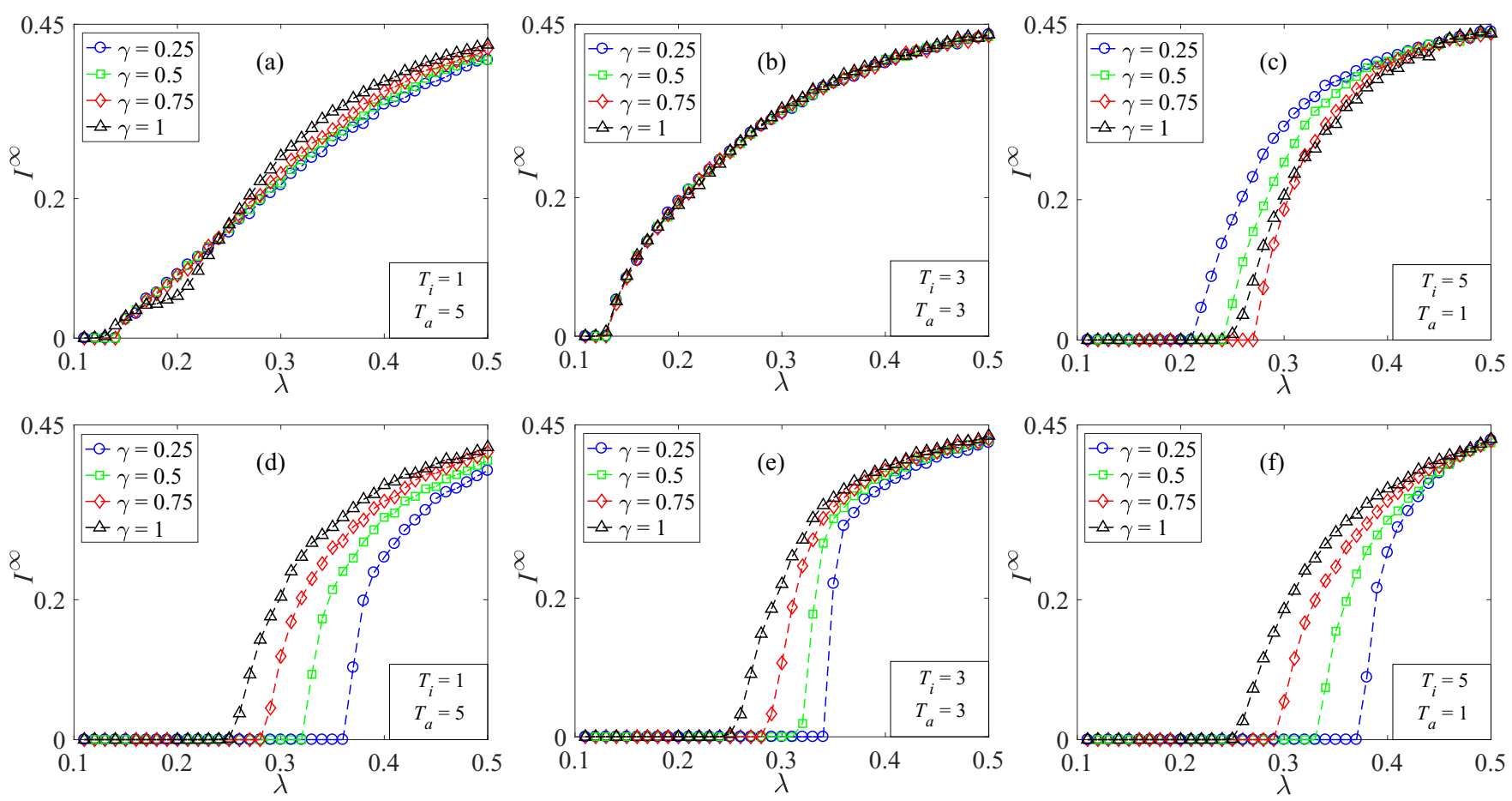

FIG. 8. Final fraction of infected nodes $I^{\infty}$ with time-varying activity as a function of infection rate $\lambda$ for three cases of threshold conditions. In the figure, (a), (b), and (c) are related to the duration $h=1$, and (d), (e), and (f) are related to the duration $h=\infty$. Other parameters are set to be the same as those in Fig. 2.

pared with $\gamma=1$, the reduction of activity has the potential to enhance the epidemic threshold and reduce the infection prevalence. Although the reduced activity of alert nodes leads to fewer susceptible-alert $(S-A)$ connections, the alert nodes are benefited with a lower infection risk whilst remaining alert due to $h=\infty$, which then facilitates awareness diffusion on the networks.

\section{CONCLUSION}

In this paper, we have investigated the effect of risk perception and awareness diffusion on the epidemic spreading process over ADNs. The risk perception is based not only on the instantaneous number of infectious neighbors, but also that of the alert neighbors. Leveraging the mean-field approximation, an SAIS model is developed incorporating the SIS disease contagion process with the awareness diffusion process characterized by a threshold. Based on the proposed model, it is shown that the information of the number of alert neighbors for risk perception and a longer duration of the preventive effect are beneficial for enhancing the epidemic threshold and reducing the infection prevalence. In addition, it can be observed from simulation that there exists a critical infection rate for triggering the cascade of awareness, which induces a two-stage characteristic for the infection prevalence. Furthermore, due to the two opposite effects embedded in the reduction in the activity of alert individuals, the interaction among the epidemic spreading, the awareness diffusion and the time-varying network structure is nontrivial.

Despite the recent burst of interest in contagion process unfolding on the time-varying networks, there is still a substantial lack of studies addressing the indirect feedback loop between the individual's behavior and epidemic spreading. Our work extends the SAIS model considered in static networks to the more accurate but demanding temporal networks. The threshold model that is more suitable for characterizing the adoption of preventive behaviors is introduced in the risk perception. Our findings unveil the important role of awareness diffusion driven by the alert individuals in temporal networks and the impact of the duration of the preventive effect. In this paper, we have considered that the awareness diffusion and epidemic spreading occur on the same network. Meanwhile, the risk perception is considered only with the information of the current epidemic situation. Therefore, the propagation of awareness and epidemic on different networks, as well as the role of historical epidemic information deserve further study in the future.

\section{ACKNOWLEDGMENTS}

This work was supported by the National Natural Science Foundation of China under Grant No. 61873194.
[1] R. Pastor-Satorras and A. Vespignani, Phys. Rev. Lett. 86, 3200 (2001).
[2] P. Van Mieghem, J. Omic, and R. Kooij, IEEE/ACM Trans. Netw. 17, 1 (2009). 
[3] C. Castellano and R. Pastor-Satorras, Phys. Rev. Lett. 105, 218701 (2010)

[4] P. Wang, M. C. González, C. A. Hidalgo, and A.-L. Barabási, Science 324, 1071 (2009).

[5] P. Holme, Phys. Rev. E 94, 022305 (2016).

[6] N. Perra, B. Gonçalves, R. Pastor-Satorras, and A. Vespignani, Sci. Rep. 2, 469 (2012).

[7] M.-X. Liu, W. Wang, Y. Liu, M. Tang, S.-M. Cai, and H.-F. Zhang, Phys. Rev. E 95, 052306 (2017).

[8] G. Ren and X. Wang, Chaos 24, 023116 (2014).

[9] M. Nadini, K. Sun, E. Ubaldi, M. Starnini, A. Rizzo, and N. Perra, Sci. Rep. 8, 2352 (2018).

[10] S.-Y. Liu, A. Baronchelli, and N. Perra, Phys. Rev. E 87, 032805 (2013).

[11] A. Sunny, B. Kotnis, and J. Kuri, Phys. Rev. E 92, 022811 (2015).

[12] M. Karsai, N. Perra, and A. Vespignani, Sci. Rep. 4, 4001 (2014).

[13] K. Sun, A. Baronchelli, and N. Perra, Eur. Phys. J. B 88, 326 (2015).

[14] I. Pozzana, K. Sun, and N. Perra, Phys. Rev. E 96, 042310 (2017).

[15] L. Alessandretti, K. Sun, A. Baronchelli, and N. Perra, Phys. Rev. E 95, 052318 (2017).

[16] K. Hoppe and G. J. Rodgers, Phys. Rev. E 88, 042804 (2013).

[17] Z. Wang, M. A. Andrews, Z.-X. Wu, L. Wang, and C. T. Bauch, Phys. Life. Rev. 15, 1 (2015).

[18] Y. Feng, L. Ding, Y.-H. Huang, and L. Zhang, Physica A 463, 493 (2016).

[19] F. D. Sahneh, A. Vajdi, J. Melander, C. Scoglio et al., IEEE Trans. Netw. Sci. Eng., 1 (2017).

[20] Q. Wu, X. Fu, M. Small, and X.-J. Xu, Chaos 22, 013101 (2012).
[21] S. Funk, E. Gilad, C. Watkins, and V. A. Jansen, Proc. Natl. Acad. Sci. U.S.A. 106, 6872 (2009).

[22] B. Kotnis and J. Kuri, Phys. Rev. E 87, 062810 (2013).

[23] A. Rizzo, M. Frasca, and M. Porfiri, Phys. Rev. E 90, 042801 (2014).

[24] A. Moinet, R. Pastor-Satorras, and A. Barrat, Phys. Rev. E 97, 012313 (2018).

[25] E. P. Fenichel, C. Castillo-Chavez, M. G. Ceddia, G. Chowell, P. A. G. Parra, G. J. Hickling, G. Holloway, R. Horan, B. Morin, C. Perrings et al., Proc. Natl. Acad. Sci. U.S.A. 108, 6306 (2011).

[26] D. J. Watts, Proc. Natl. Acad. Sci. U.S.A. 99, 5766 (2002).

[27] Z. Ruan, G. Iniguez, M. Karsai, and J. Kertész, Phys. Rev. Lett. 115, 218702 (2015).

[28] H.-X. Yang, M. Tang, and Z. Wang, Phys. A 490, 347 (2018).

[29] A. Czaplicka, R. Toral, and M. San Miguel, Phys. Rev. E 94, 062301 (2016).

[30] D. Centola, Science 329, 1194 (2010).

[31] A. Nematzadeh, E. Ferrara, A. Flammini, and Y.-Y. Ahn, Phys. Rev. Lett. 113, 088701 (2014).

[32] F. Karimi and P. Holme, Phys. A 392, 3476 (2013).

[33] V.-P. Backlund, J. Saramäki, and R. K. Pan, Phys. Rev. E 89, 062815 (2014).

[34] C. Granell, S. Gómez, and A. Arenas, Phys. Rev. E 90, 012808 (2014).

[35] Q. Guo, X. Jiang, Y. Lei, M. Li, Y. Ma, and Z. Zheng, Phys. Rev. E 91, 012822 (2015).

[36] Q. Guo, Y. Lei, X. Jiang, Y. Ma, G. Huo, and Z. Zheng, Chaos 26, 043110 (2016).

[37] M. E. J. Newman, Phys. Rev. Lett. 89, 208701 (2002).

[38] F. D. Sahneh, F. N. Chowdhury, and C. M. Scoglio, Sci. Rep. 2, 632 (2012).

[39] M. Starnini and R. Pastor-Satorras, Phys. Rev. E 87, 062807 (2013). 